\title{
Bound states in the dynamics of a dipole in the presence of a conical defect
}

\author{
C. A. de Lima Ribeiro \\ Departamento de Física - Universidade Estadual de Feira de Santana \\ BR 116-Norte, Km 3, 44.031-460 - Feira de Santana, BA, Brazil \\ Claudio Furtado \\ Departamento de Física-Universidade Federal da Paraíba, \\ Caixa Postal 5008, 58.051-970 - João Pessoa, PB, Brazil \\ Fernando Moraes \\ Laboratório de Física Teórica e Computacional \\ Departamento de Física-Universidade Federal de Pernambuco \\ 50.670-901 - Recife, PE, Brazil and \\ Departamento de Física-Universidade Federal da Paraíba, \\ Caixa Postal 5008, 58.051-970 - João Pessoa, PB, Brazil
}

\begin{abstract}
In this work we investigate the quantum dynamics of an electric dipole in a $(2+1)$-dimensional conical spacetime. For specific conditions, the Schrödinger equation is solved and bound states are found with the energy spectrum and eigenfunctions determined. We find that the bound states spectrum extends from minus infinity to zero with a point of accumulation at zero. This unphysical result is fixed when a finite radius for the defect is introduced.
\end{abstract}

PACS numbers: 11.27.+d, 03.65.Ge, 04.20.-q

Lower-dimensional gravity, besides its pedagogical role [1], has important applications in condensed matter physics: elastic solids with defects can be mapped into three-dimensional gravity with torsion [2]. Within this framework, we have studied [3, 4, 5] a few cases concerning classical and quantum effects in solid media containing topological defects such as disclinations and dislocations (a disclination in a solid is analogous to a cosmic string in spacetime). In particular, the scattering states of a single electric dipole in a (2+1)-dimensional conic space-time was investigated in [6]. From a different point of view, the dipole dynamics was also studied in [7]. An important issue in this class of problems is the question of the self-interaction of a point charge in the background space of a topological defect [8, 9, 10, 11]. Due to its simplicity the cosmic string provides a prototype spacetime for the investigation of the non-local influence of gravity on matter fields. The effect is usually studied in the spacetime of a single cosmic string, but multiple cosmic strings and multiconical space-time have also been considered [12]. For simplicity, we restrain our study to the $(2+1)$-dimensional section of the cosmic string metric

$$
d s^{2}=-c^{2} d t^{2}+d \rho^{2}+\frac{\rho^{2}}{p^{2}} d \theta^{2},
$$

in polar coordinates. The linear mass density $\mu$ is related to the parameter $p$ by $p=(1-4 G \mu)^{-1}>1$, where $G$ is the gravitational constant. This dimensional reduction can be justified by invoking translational invariance along the string axis.

In this letter, the study of the bound states of an electric dipole to a $(2+1)$-dimensional conical singularity is analyzed. Treating the self-interaction by a classical field theory approach [13], the self-energy is obtained and so is the Schrödinger equation in this background. By solving the Schrödinger equation the energy spectrum is found.

The effective interaction between the dipole and the defect is the electromagnetic self-energy of the dipole due to the non-flat background created by the defect. For instance, a point charge in a static gravitational field experiences an electrostatic force due to the deformation of its electric field lines produced by the particular geometry of spacetime [14]. The net effect is a self-force on the charge. A straightforward method to calculate the self-energy in a $(2+1)$-dimensional space-time has been developed by Grats and Garcia [13]. The regularized self-energy is therefore found to be

$$
U=\frac{\left(p^{2}-1\right)}{48 \pi} \frac{D^{2} \cos (2 \phi)}{\rho^{2}},
$$

where $\phi$ is the angle between a vector directed from the singularity and the dipole direction and $D$ is the dipole moment.

Notice that a minimum for the self-energy is obtained when $\phi=\frac{\pi}{2}$. From this it is seen that deflections from equilibrium position leads to a restorative force which tends to align the dipole perpendicular to the vector directed 
from the singularity. This rotational degree of freedom will have a small contribution for the overall dynamics of the dipole, in particular if we are looking at low or null rotational angular momentum states. Therefore, from now on we will not consider this degree of freedom; that is, we take

$$
U=-\frac{\left(p^{2}-1\right) D^{2}}{48 \pi \rho^{2}}
$$

We thus have an attractive inverse square potential. This kind of potential has attracted substantial attention recently. It was extensively studied by Case [15] in 1950 and more recently by a number of authors [16, 17]. Recently, Audretsch et al. [18 studied a similar potential in the context of the Aharonov-Bohm scattering problem where the bound states are possible solutions.

With the kinetic energy given in terms of the Laplace-Beltrami operator

$$
\nabla^{2}=\frac{1}{\rho} \partial_{\rho}\left(\rho \partial_{\rho}\right)+\frac{p^{2}}{\rho^{2}} \partial_{\theta \theta}
$$

we arrive at the following Schrödinger equation

$$
\left[\frac{1}{\rho} \partial_{\rho}\left(\rho \partial_{\rho}\right)+\frac{p^{2}}{\rho^{2}} \partial_{\theta \theta}+\frac{2 m \mathcal{E}}{\hbar^{2}}+\frac{\left(p^{2}-1\right) d^{2}}{48 \pi \rho^{2}}\right] \Psi(\rho, \theta)=0,
$$

where $d$ is the normalized dipole moment, given by $d^{2}=\frac{2 m}{\hbar^{2}} D^{2}$.

Considering rotational symmetry we write the solution in the form

$$
\Psi(\rho, \theta)=R(\rho) \exp i l \theta
$$

where $l$ is the orbital angular momentum quantum number. This leads to the Bessel equation

$$
R^{\prime \prime}(\rho)+\frac{1}{\rho} R^{\prime}(\rho)+\left[\frac{\nu^{2}}{\rho^{2}}-k^{2}\right] R(\rho)=0,
$$

with

$$
k^{2}=-\frac{2 m \mathcal{E}}{\hbar^{2}}
$$

and $\nu$ given by

$$
\nu^{2}=\frac{\left(p^{2}-1\right) d^{2}}{48 \pi}-(l p)^{2} .
$$

Notice that the condition which may lead to bound state solutions depends on the balance between the electric dipole intensity $d$ and the angular momentum corrected by the parameter $p$. So, we are interested in those low angular momentum states where $\nu^{2}>0$.

Now we do the following transformation in the radial equation (7):

$$
S(\rho)=\rho^{\frac{1}{2}} R(\rho) .
$$

The radial equation is transformed to

$$
S^{\prime \prime}(\rho)+\left[\frac{\nu^{2}+\frac{1}{4}}{\rho^{2}}-k^{2}\right] S(\rho)=0,
$$

Also, for convenience, we make $\rho=x / k$ and $S(\rho)=S(x / k)=u(x)$ where $x$ is a adimensional quantity. Eq. (11) becomes now

$$
u^{\prime \prime}(x)+\left[\frac{\nu^{2}+\frac{1}{4}}{x^{2}}-1\right] u(x)=0,
$$

where the derivatives are now taken with respect to $x$. The solution to equation (12) is given in terms of the modified Bessel function of third kind and imaginary order $K_{i \nu}(x)$ [19]

$$
u(x)=\sqrt{x} K_{i \nu}(x),
$$


with the range $[k a, \infty]$, where $a$ is the radius of the string. Therefore, we require

$$
u(k a)=\sqrt{k a} K_{i \nu}(k a)=0 .
$$

In order to find the zeros of the Bessel function $K_{i \nu}(x)$ we look at the series expansion [20]

$$
K_{i \nu}(x)=\sum_{j=0}^{\infty} c_{j} f_{j}
$$

where

$$
c_{j}=\left(\frac{x^{2}}{4}\right)^{j} \frac{1}{k !}
$$

and

$$
f_{j}=\frac{\pi}{2 \sin (i \nu \pi)}\left[\frac{(x / 2)^{-i \nu}}{\Gamma(j+1-i \nu)}-\frac{(x / 2)^{i \nu}}{\Gamma(j+1+i \nu)}\right] .
$$

The expansion is justified because of the smallness of the string radius and also because $p$ is very close to 1 .

Now, the above equations (16) and (17) yield

$$
\begin{aligned}
& c_{0}=1, \\
& f_{0}=\sqrt{\frac{\pi}{\nu \sinh (\pi \nu)}} \sin [\nu \ln (x / 2)+\nu \gamma],
\end{aligned}
$$

where $\gamma$ is Euler's constant. This leads to the following expansion

$$
K_{i \nu}(x) \approx \sqrt{\frac{\pi}{\nu \sinh (\pi \nu)}} \sin [\nu \ln (x / 2)+\nu \gamma]+O(\nu) .
$$

Therefore, for small $x$ and $\nu$, the zeros of the Bessel $K_{i \nu}(x)$ function are given by

$$
x_{n}=2 e^{-n \pi / \nu-\gamma}[1+O(\nu)]
$$

where, $n=1,2, \ldots, \infty$. From $k a=x_{n}$ and from equation (8) we get the energy levels

$$
\mathcal{E}=-\frac{2 \hbar^{2} e^{-2 n \pi / \nu-2 \gamma}}{m a^{2}}[1+O(\nu)]
$$

Notice that the spectrum has a point of accumulation at zero and that, if the radius $a$ of the string is taken to zero there, is no lower bound for the energy levels, which will range from minus infinity to zero. Fortunately, a physical string must have a non-vanishing core, providing a natural regularization for this problem.

In summary, in this work we analyze a non-relativistic problem in which an electric dipole interacts with a cosmic string via a $1 / \rho^{2}$ effective potential. We have shown that this potential under a specific condition leads to bound states. This potential is nevertheless pathological in the sense that the bound states have no lower bound, ranging from minus infinity to zero, making these bound states unstable. This difficulty comes from the assumption of an infinitely thin cosmic string. Actually, a more realistic model for the cosmic string presumes a finite string radius. This provides a natural cutoff for the $1 / \rho^{2}$ singularity and gives a well defined energy spectrum for the problem. Finally, the study of the spectrum of dipoles bound to a cosmic string may be a useful tool for the interpretation of astrophysical data in the search for cosmic strings.

\section{Acknowledgments}

We would like to thank CNPq, CAPES (PROCAD), FAPESB and PROINPE from Universidade Estadual de Feira de Santana for financial supports.

[1] R. Jackiw Nucl. Phys. B 252, 343 (1985); S. Deser, R. Jackiw and G. 'tHooft Ann. Phys. (N.Y.) 152, 220 (1984).

[2] M.O. Katanaev and I.V. Volovich, Ann. Phys. (NY) 216, 1 (1992). 
[3] C. Furtado and F. Moraes, Phys. Lett. 188A, 394 (1994).

[4] S. Azevedo and F. Moraes, J. Phys.: Cond. Matt. 12, 7421 (2000).

[5] C. Furtado and F. Moraes, J. Phys. A: Math. Gen. 33, 5513 (2000).

[6] C. A. de Lima Ribeiro, C. Furtado, V. B. Bezerra, and F. Moraes, J. Phys. A:Math Gen. 34, 6119 (2001).

[7] G. Spavieri, Phys. Rev. A 59, 3194 (1999).

[8] B. Linet, Phys. Rev. D 33, 1833 (1986).

[9] A. G. Smith Proc. Symp. on the Formation and Evolution of Cosmic Strings ed G. W. Gibbons, S. W. Hawking and T. Vachaspati (Cambridge: Cambridge University Press).

[10] T Souradeep and V. Sahni, Phys Rev. D 46, 1616 (1992).

[11] E. R. Bezerra de Mello, V. B. Bezerra, C. Furtado and Fernando Moraes, Phys. Rev. D 51, 7140 (1995).

[12] E.R.B. de Mello, V.B. Bezerra and Yu V. Grats, Class. Quantum Grav. 15, 1915 (1998).

[13] Y. Grats and A. Garcia, Class. Quantum Grav. 13, 189 (1996).

[14] C. M. de Witt and B. S. de Witt, Physics 1, 3 (1964); W. G. Unrunh, Proc. R. Soc. London A 348, 447 (1976).

[15] K.M. Case, Phys. Rev. 80, 797 (1950).

[16] H. E. Camblong et al., Phys. Rev. Lett. 85, 1590 (2000) and references therein.

[17] K.S. Gupta and S.G. Rajeev, Phys. Rev. 48, 5940 (1993).

[18] J. Audretsch, V.D. Skarzhinsky and B.L. Voronov, J. Phys. A: Math.and Gen. 34, 235 (2001)

[19] G.N. Watson, Theory of Bessel Functions (The Macmillan Company, New York, 1944) p.77.

[20] A. Gil, J. Segura and N.M. Temme, J. Comput. Phys 175, 398 (2002). 